\title{
Endoplasmic Reticulum Stress Induces MUC5AC and MUC5B Expression in Human Nasal Airway Epithelial Cells
}

\author{
Min Han Kim ${ }^{1, *}$ (i) $\cdot$ Chang Hoon Bae ${ }^{1, \star} \cdot$ Yoon Seok Choi ${ }^{1} \cdot$ Hyung Gyun $\mathrm{Na}^{1} \cdot$ Si-Youn Song ${ }^{1}$ Yong-Dae Kim ${ }^{1,2}$ (1) \\ ${ }^{1}$ Department of Otorhinolaryngology-Head and Neck surgery, College of Medicine, Yeungnam University, Daegu; \\ ${ }^{2}$ Regional Center for Respiratory Diseases, Yeungnam University Medical Center, Daegu, Korea
}

Objectives. Endoplasmic reticulum (ER) stress is known to be associated with inflammatory airway diseases, and three major transmembrane receptors: double-stranded RNA-activated protein kinase-like ER kinase, inositol requiring enzyme 1, and activating transcription factor 6 (ATF6) play important roles in ER stress-related proinflammatory signaling. However, the effects of ER stress and these three major signaling pathways on the regulation of the production of airway mucins in human nasal airway epithelial cells have not been elucidated.

Methods. In primary human nasal epithelial cells, the effect of tunicamycin (an ER stress inducer) and 4-phenylbutyric acid (4-PBA, ER stress inhibitor) on the expression of MUC5AC and MUC5B was investigated by reverse transcriptasepolymerase chain reaction, real-time polymerase chain reaction, enzyme immunoassay, and immunoblot analysis. Small interfering RNA (siRNA) transfection was used to identify the mechanisms involved.

Results. Tunicamycin increased the expressions of MUC5AC and MUC5B and the mRNA expressions of ER stress-related signaling molecules, including spliced X-box binding protein 1 (XBP-1), transcription factor CCAAT-enhancer-binding protein homologous protein (CHOP), and ATF6. In addition, 4-PBA attenuated the tunicamycin-induced expressions of MUC5AC and MUC5B and the mRNA expressions of ER stress-related signaling molecules. Furthermore, siRNA knockdowns of XBP-1, CHOP, and ATF6 blocked the tunicamycin-induced mRNA expressions and glycoprotein productions of MUC5AC and MUC5B.

Conclusion. These results demonstrate that ER stress plays an important role in the regulation of MUC5AC and MUC5B via the activations of XBP-1, CHOP, and ATF6 in human nasal airway epithelial cells.

Keywords. Endoplasmic Reticulum Stress; X-Box Binding Protein 1; Transcription Factor CHOP; Activating Transcription Factor 6; Mucins

\section{INTRODUCTION}

Endoplasmic reticulum (ER) stress response is an important phenomenon observed in many inflammatory diseases, and re-

\footnotetext{
- Received April 3, 2018

Revised September 4, 2018

Accepted September 9, 2018

- Corresponding author: Yong-Dae Kim

Department of Otorhinolaryngology-Head and Neck Surgery,

College of Medicine, Yeungnam University, 170 Hyeonchung-ro,

Nam-gu, Daegu 42415, Korea

Tel: +82-53-620-3781, Fax: +82-53-628-7884

E-mail: ydkim@med.yu.ac.kr

*These authors contributed equally to this work.
}

sults in the activation of unfolded protein response [1,2]. Moreover, ER stress response has been implicated in the pathogeneses of various inflammatory airway diseases, including viral and bacterial infections, cystic fibrosis, asthma, and chronic obstructive pulmonary disease [2-4]. Eukaryotic cells exhibit three major ER stress pathways, that is the double-stranded RNA-activated protein kinase-like ER kinase (PERK), inositol requiring enzyme 1 (IRE1) involved in X-box binding protein 1 (XBP-1), and the activating transcription factor 6 (ATF6) pathways. Furthermore, these three major pathways function differently in ways determined by the pathogeneses of airway diseases [3-7].

The surfaces of the human nasal mucosa are continuously ex-

Copyright @ 2019 by Korean Society of Otorhinolaryngology-Head and Neck Surgery.

This is an open-access article distributed under the terms of the Creative Commons Attribution Non-Commercial License (http://creativecommons.org/licenses/by-nc/4.0)

which permits unrestricted non-commercial use, distribution, and reproduction in any medium, provided the original work is properly cited. 
posed to particulates and pathogens. Human nasal airway epithelial cells secrete mucus which serves as the first line of defense against inhaled pathogens as protective mucus barriers trap and allow the elimination of pathogens via mucociliary clearance [8-10]. However, excessive mucus is major symptom, which is associated with goblet cell metaplasia and hypertrophy of nasal airway epithelium in chronic rhinosinusitis (CRS). Mucins are high molecular weight glycoproteins, one of major component responsible for mucus secretion, impart different physical and biological properties to mucus of the airway [11,12]. Among the airway mucins, the levels of MUC5B and MUC5AC mRNA in CRS were significantly increased compared with those in normal sinus mucosa. This change of nasal mucin gene expression by sinus epithelium as a consequence of sinus disease will almost certainly affect the composition and biophysical properties of sinus mucus [8]. Nevertheless, the mechanisms underlying overproduction of MUC5B and MUC5AC glycoprotein in CRS are not fully understood.

Accordingly, our aims were to determine whether ER stress affects regulation of the expressions of MUC5AC and MUC5B and to investigate the signaling pathways responsible for the ER stress-induced expressions of MUC5AC and MUC5B in human nasal airway epithelial cells.

\section{MATERIALS AND METHODS}

\section{Materials}

Tunicamycin (an ER stress inducer) and 4-phenylbutyric acid (4PBA, an ER stress inhibitor) were obtained from Sigma-Aldrich (St. Louis, MO, USA). Primary human nasal epithelial cells (HNEpCs) and airway epithelial cell growth medium were purchased from PromoCell (Heidelberg, Germany). RPMI 1640 medium and Trizol were from Invitrogen (Carlsbad, CA, USA). EZ-Cytox Cell Viability Assay kits were purchased from Daeil Laboratories (Seoul, Korea). Reverse transcriptase-polymerase chain reaction (RT-PCR) kits were from Applied Biosystems (Foster City, CA, USA). Real-time PCR kits were obtained from Roche Applied Science (Mannheim, Germany). Primary and secondary antibodies for MUC5AC and MUC5B (used immunoassay) were obtained from Santa Cruz Biotechnology (Santa Cruz, CA, USA). ForWestern blot analysis, primary and secondary antibodies of XBP-1, CCAAT-enhancer-binding protein ho-

\section{H I}

- Endoplasmic reticulum (ER) stress induces the expression of MUC5AC and MUC5B in human nasal airway epithelial cells.

- The expression of secretary mucins is induced by ER stress via multiple signaling mechanisms. mologous protein (CHOP), ATF6 and $\beta$-actin were purchased from Abcam (Cambridge, MA, USA). Fetal bovine serum (FBS) was purchased from Hyclone Laboratories (Logan, UT, USA). OPTI-MEM I Reduced Serum Medium, Lipofectamine 2000, predesigned small interfering RNAs (siRNAs) targeting XBP-1, CHOP, and ATF6, and control siRNA were purchased from Thermo Fisher Scientific (Lafayette, CO, USA). This study was approved by the Institutional Review Board of Yeungnam University Medical Center (IRB No. YUMC 2015-06-058).

\section{Cell culture and treatment}

HNEpCs were cultured in Airway Epithelial Cell Growth Medium at $37^{\circ} \mathrm{C}$ in $5 \% \mathrm{CO}_{2}$ fully humidified air and subcultured according to the recommendations of PromoCell. Passage-2 HNEpCs $\left(1 \times 10^{5}\right.$ cell/culture) were seeded in $0.5 \mathrm{~mL}$ of culture medium. When the cultures were confluent, the cells were exposed to the indicated concentrations of tunicamycin or 4-PBA. The proliferations and viabilities of HNEpCs were quantified using an EZ-Cytox Cell Viability Assay kit, and changes in cell morphology were documented using a microscope.

\section{RT-PCR of the mRNA expressions of MUC5AC, MUC5B, spliced XBP-1, CHOP, and ATF6}

Total RNA was isolated from cultured cells using RT-PCR kits (Applied Biosystems). Samples were reverse transcribed into complementary deoxyribonucleic acid (cDNA) using the GeneAmp RNA PCR core kit (Applied Biosystems). The primer sequences and conditions used for MUC5AC, MUC5B, spliced XBP-1, CHOP, and ATF6 were as previously described protocols (Table 1) $[6,13,14]$. RNA integrities and the success of RT reactions were monitored by PCR amplification of transcripts using of the glyceraldehyde-3-phosphate dehydrogenase (GAPDH) gene. PCR products were electrophoresed in $2 \%$ agarose gels, and visualized by staining with ethidium bromide and ultraviolet fluorescence. RT-PCR products were semiquantitatively analyzed using scanned gel images and by measuring the intensities of PCR products using commercially available imaging software (Scion, Frederick, MD, USA). Relative intensities were calculated by expressing the intensities of MUC5AC, MUC5B, spliced XBP-1, CHOP, and ATF6 as ratios of GAPDH intensities.

\section{Real-time PCR for the mRNA expressions of MUC5AC, MUC5B, spliced XBP-1, CHOP, and ATF6}

Real-time PCR was performed using the LightCycler FastStart DNA Master SYBR Green kit (Roche Applied Science) and $0.5 \mu \mathrm{L}$ of cDNA (corresponding to $25 \mathrm{ng}$ of total RNA) in a final volume of $10 \mu \mathrm{L}$ of $2.5 \mathrm{mM} \mathrm{MgCl}_{2}$ and $0.5 \mu \mathrm{M}$ of each primer (final concentration). Quantitative PCR was performed using a LightCycler using 45 cycles of $95^{\circ} \mathrm{C}$ for 10 seconds, a specific annealing temperature for 5 seconds, and $72^{\circ} \mathrm{C}$ for 10 seconds. Data were normalized versus GAPDH. Melting curves were used to evaluate amplification specificity, according to the man- 
Table 1. Primer sequences, lengths of amplified templates, and primer-specific conditions for RT-PCR

\begin{tabular}{|c|c|c|c|c|}
\hline Gene & Primer sequence (5'-3') & Product (bp) & Cycle & Annealing temperature $\left({ }^{\circ} \mathrm{C}\right)$ \\
\hline MUC5AC & $\begin{array}{l}\text { Forward: TCC ACC ATA TAC CGC CAC AGA } \\
\text { Reverse: TGG ACG GAC AGT CAC TGT CAA C }\end{array}$ & 103 & 30 & 60 \\
\hline MUC5B & $\begin{array}{l}\text { Forward: CAC ATC CAC CCT TCC AAC } \\
\text { Reverse: GGC TCA TTG TCG TCT CTG }\end{array}$ & 245 & 30 & 60 \\
\hline$s X B P-1$ & $\begin{array}{l}\text { Forward: TAC GGG AGA AAA CTC ACG GC } \\
\text { Reverse: TTC CAG CTT GGC TGA TGA GG }\end{array}$ & 421 & 40 & 58 \\
\hline $\mathrm{CHOP}$ & $\begin{array}{l}\text { Forward: CCT TCC AGT GTG TGG GAC Tा } \\
\text { Reverse: GTG TGT TाT CCT TाT GCC GT }\end{array}$ & 259 & 30 & 60 \\
\hline ATF6 & $\begin{array}{l}\text { Forward: CAA CCC ACG TTG TCA GCA CCA TC } \\
\text { Reverse: ACA GTT CTC TGC CTG CCA CCA AG }\end{array}$ & 143 & 30 & 65 \\
\hline
\end{tabular}

RT-PCR, reverse transcriptase-polymerase chain reaction.

ufacturer's instructions (Roche Applied Science).

\section{Enzyme-linked immunosorbent assay analysis of MUC5AC and MUC5B glycoproteins}

The protein levels of MUC5AC and MUC5B were determined by enzyme-linked immunosorbent assay (ELISA). Samples of cell supernatants were prepared in phosphate-buffered saline (PBS) at several dilutions, incubated at $40^{\circ} \mathrm{C}$ in a 96 -well plate until dry, washed three times with PBS, blocked with $2 \%$ FBS for 1 hour at room temperature, rewashed three times with PBS, and incubated with primary antibody for MUC5AC or MUC5B (diluted at 1:200 with PBS containing 0.05\% Tween 20) for 1 hour. Wells were then washed three times with PBS, and horseradish peroxidase-conjugated secondary antibody for MUC5AC or MUC5B was added to wells, and allowed to stand for 4 hours. Color was developed using 3,3',5,5'-tetramethylbenzidine peroxidase solution and stopped with $2 \mathrm{~N}-\mathrm{H}_{2} \mathrm{SO}_{4}$. Optical densities were measured using an ELISA reader (EL800; BIO-TEK Instruments, Winooski, VT, USA) at $450 \mathrm{~nm}$. Results are expressed as percentages of baseline controls.

\section{Western blot analysis for XBP-1, CHOP, and ATF6}

HNEpCs were seeded in each well of a 6-well plate, and treated with the indicated concentrations of tunicamycin. The cells harvested $200 \mu \mathrm{L}$ radioimmunoprecipitation assay buffer with phosphatase inhibitor cocktail and incubated for 20 minutes at $4^{\circ} \mathrm{C}$. And then sample were centrifuged at 2,500 $\times g$ for 10 minutes at $4^{\circ} \mathrm{C}$ and the supernatant (whole-cell lysate) was collected. Proteins $(20 \mu \mathrm{g})$ were separated using $10 \%$ reducing sodium dodecyl sulfate-polyacrylamide gel electrophoresis and electroblotted onto a polyvinylidene difluoride membrane. The membrane was blocked $5 \%$ bovine serum albumin in tris buffered saline with $0.1 \%$ Tween 20 , followed by incubation with the indicated primary antibody of XBP-1 (ab37152), CHOP (ab11419) and ATF6 (ab37149) for 4 hours. Subsequently, the membrane was incubated for 1 hour with secondary antibody of XBP-1, CHOP and ATF6 conjugated to horseradish peroxidase, and developed using an enhanced chemiluminescence system kit. Signals correspond- ing to the protein of interest were developed using an enhanced West Pico Chemiluminescent Substrate (Thermo Fisher, Boston, MA, USA). Bands were detected after exposure of the membrane to an imaging system (Chemiluminescence image system, FUSION-FX7 820wL, Vilber, Eberhardzell, Germany).

\section{Cell transfection with siRNAs for XBP-1, CHOP, or ATF6}

The transfection rate of each siRNA was confirmed to be $\geq 90 \%$. Transfection was performed according to the manufacturer's instructions (Thermo Fisher Scientific); XBP-1 siRNA (s14913), CHOP (VHS40605), and ATF6 (s223543). In brief, HNEpCs were seeded in the wells of 6-well plates at $1 \times 10^{5}$ cells/well and incubated overnight in RPMI 1640 medium. When cells were $80 \%-90 \%$ confluent, the following day, they were washed with PBS and OPTI-MEM I Reduced Serum Medium was added. Separately, XBP-1 siRNA and Lipofectamine 2000 were incubated together in OPTI-MEM I Reduced Serum Medium to form a XBP-1 siRNA-Lipofectamine complex, and this complexcontaining medium was then added to wells containing the cells, which were then incubated for 24 hours at $37^{\circ} \mathrm{C}$ in a $\mathrm{CO}_{2}$ incubator. The XBP-1 siRNA-Lipofectamine complex-containing medium was then replaced with RPMI 1640 medium after 4 hours without loss of transfection activity. After 24 hours of transfection with XBP-1 siRNA, cells were exposed to tunicamycin $(0.5 \mu \mathrm{g} / \mathrm{mL})$ and then harvested for RT-PCR analysis. The transfection rate of XBP-1 siRNA was confirmed to be $\geq 90 \%$. The same procedure was used to transfect cells with the siRNAs for CHOP or ATF6.

\section{Statistical analysis}

Statistical analysis was performed using the SPSS ver. 12.0 (SPSS Inc., Chicago, IL, USA). The mean and standard deviation were calculated for sets of measurements. Data comparison was done with the unpaired $t$-test or Kruskal-Wallis one-way analysis of variance followed by the Mann-Whitney test. For all tests, a $P$ value $<0.05$ was considered statistically significant. 

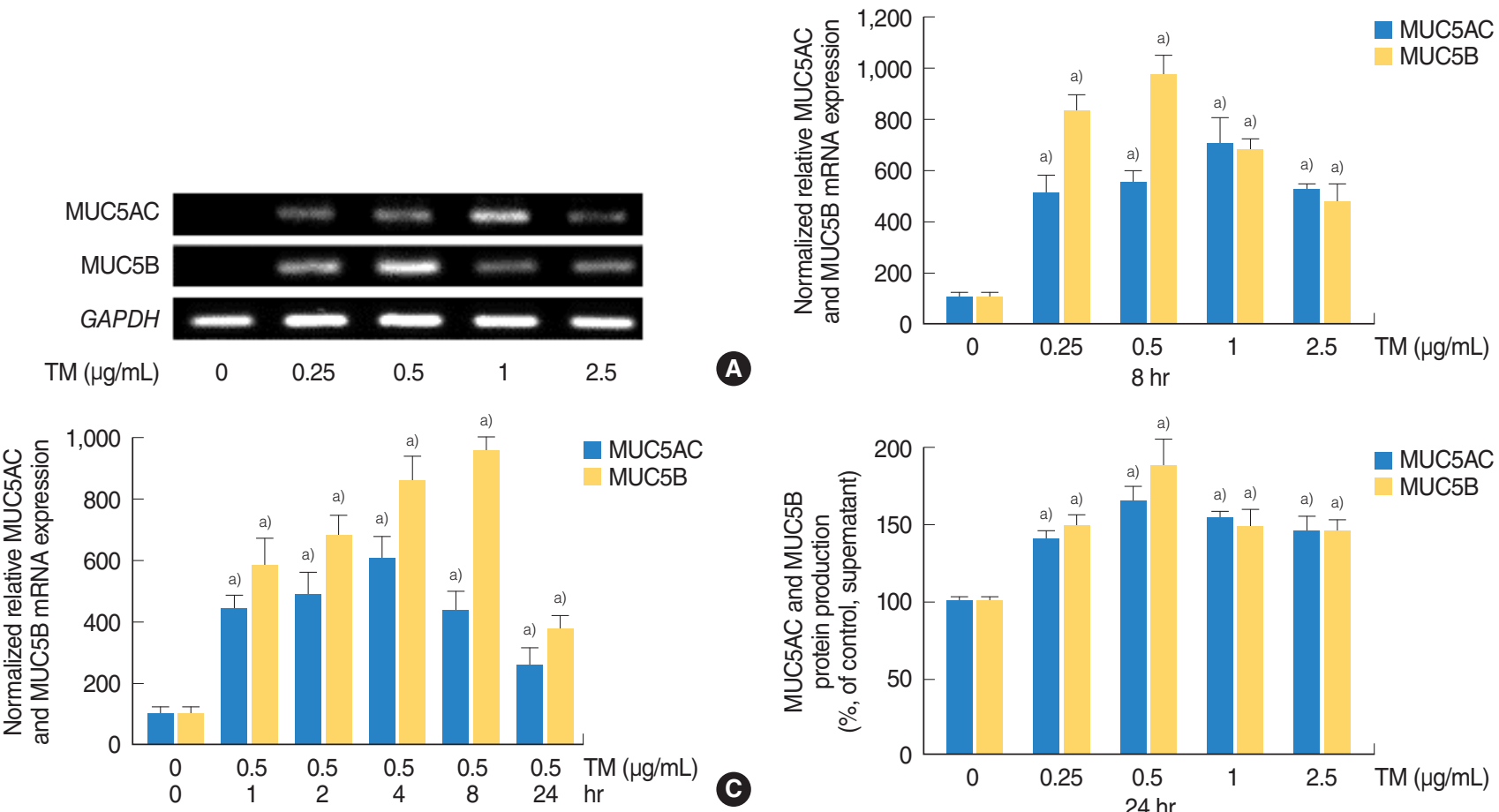

B
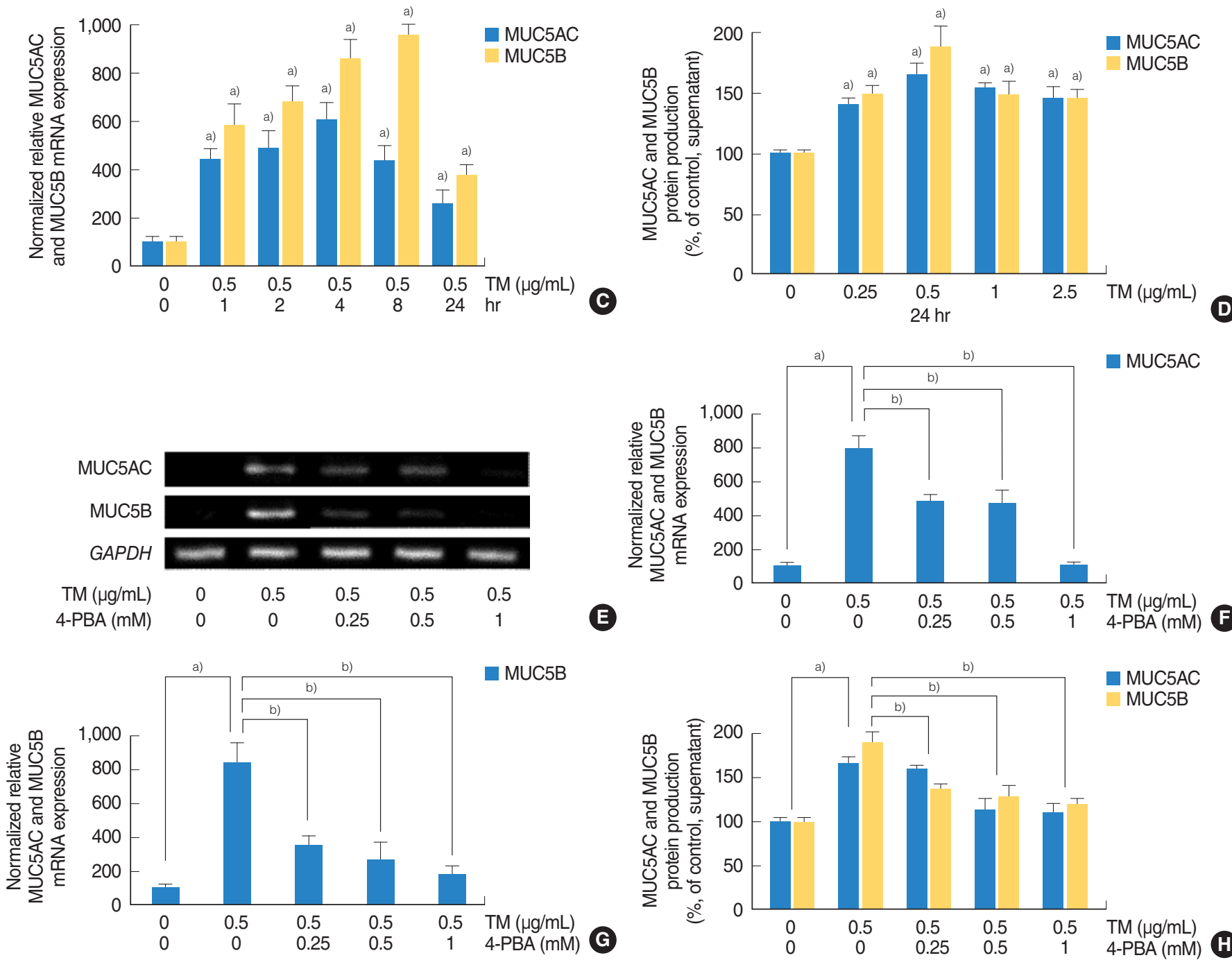

Fig. 1. Effects of tunicamycin (TM; an endoplasmic reticulum stress inducer) on the expressions of MUC5AC and MUC5B in human nasal epithelial cells. (A, B) Reverse transcriptase-polymerase chain reaction (RT-PCR) and real-time PCR showed that TM significantly increased the mRNA expressions of MUC5AC and MUC5B at all concentrations examined. (C) Time-dependent changes, real-time PCR showed that TM induced the expressions of MUC5AC and MUC5B mRNA at all times. However, peak times differed; MUC5AC peaked after 4 hours of exposure to TM, whereas MUC5B peaked after 8 hours of exposure. (D) Enzyme-linked immunosorbent assay (ELISA) showed TM induced the productions of MUC5AC and MUC5B glycoproteins. Effects of 4-phenylbutyric acid (4-PBA; an endoplasmic reticulum stress inhibitor) on the TM-induced expressions of MUC5AC and MUC5B in human nasal epithelial cells. (E-G) RT-PCR and real-time PCR showed that 4-PBA significantly attenuated the TM-induced expressions of MUC5AC and MUC5B mRNA. (H) ELISA also showed that 4-PBA significantly attenuated TM-induced the productions of MUC5AC and MUC5B glycoprotein. The images shown are representative of three separate experiments performed in triplicate. Bars represent the averages \pm standard deviation of three independent experiments performed in triplicate. GAPDH, glyceraldehyde-3-phosphate dehydrogenase. ${ }^{\text {a) }} P<0.05$ vs. baseline. ${ }^{\text {b) }} P<0.05$ vs. TM $(0.5 \mu \mathrm{g} / \mathrm{mL})$ alone. 


\section{RESULTS}

\section{ER stress increased the expressions of MUC5AC and} MUC5B in HNEpCs

HNEpCs were incubated with tunicamycin $(0,0.25,0.5,1$, or $2.5 \mu \mathrm{g} / \mathrm{mL}$ ) for 8 hours and then RT-PCR and real-time PCR were performed to determine the expressions of MUC5AC and MUC5B. The results obtained showed that tunicamycin significantly induced the mRNA expression of MUC5AC and MUC5B
(Fig. 1A, B). To determine changes in the mRNA expressions of MCU5AC and MUC5B after different exposure times, HNEpCs were treated with tunicamycin $(0.5 \mu \mathrm{g} / \mathrm{mL})$ for various times $(0$, $1,2,4,8$, or 24 hours). Real-time PCR showed that tunicamycin induced the expressions of MUC5AC and MUC5B at all times, but peak expression times differed; the mRNA expression level of MUC5AC peaked after 4 hours of exposure to tunicamycin, whereas the mRNA expression level of MUC5B peaked at 8 hours (Fig. 1C). ELISA also showed tunicamycin induced the
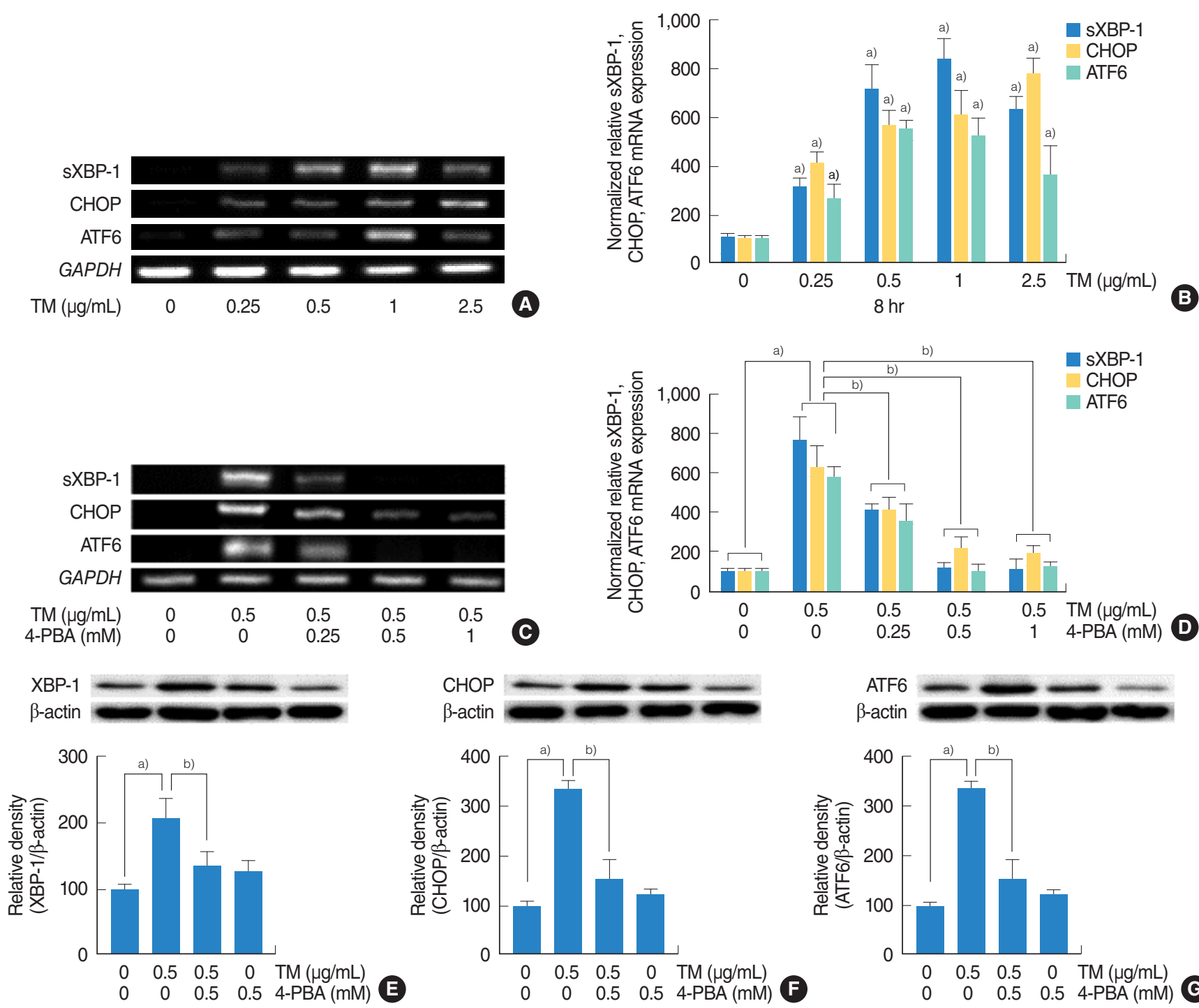

Fig. 2. The roles of the three major endoplasmic reticulum stress-mediated signaling pathways (IRE1/XBP-1, PERK/ATF4/CHOP, and ATF6) on the expressions of MUC5AC and MUC5B in human nasal epithelial cells. (A-D) Reverse transcriptase-polymerase chain reaction (RT-PCR) and real-time PCR showed that the mRNA expressions of sXBP-1, CHOP, or ATF6 were significantly increased at all doses of tunicamycin (TM; $0.25,0.5,1,2.5 \mu \mathrm{g} / \mathrm{mL}$ ) administered and that these increases were attenuated by 4-phenylbutyric acid (4-PBA). (E-G) Western blot also showed that TM induced XBP-1, CHOP and ATF6 and attenuated by 4-PBA. The images shown are representative of three separate experiments performed in triplicate. Bars represent the averages \pm standard deviation of three independent experiments performed in triplicate. sXBP-1, spliced X-box binding protein 1; CHOP, CCAAT-enhancer-binding protein homologous protein; ATF6, activating transcription factor 6; GAPDH, glyceraldehyde-3-phosphate dehydrogenase; XBP-1, X-box binding protein 1; IRE1, inositol requiring enzyme 1; PERK, RNA-activated protein kinase-like endoplasmic reticulum kinase; ATF4, activating transcription factor 4 . ${ }^{\text {a) }} P<0.05 \mathrm{vs}$. baseline. ${ }^{\text {b) }} P<0.05 \mathrm{vs}$. TM $(0.5 \mu \mathrm{g} / \mathrm{mL})$ alone. 
productions of MUC5AC and MUC5B glycoproteins (Fig. 1D).

\section{4-PBA reduced tunicamycin-induced MUC5AC and MUC5B expressions in HNEpCs}

To confirm the effect of tunicamycin (an ER stress inducer) on MUC5AC and MUC5B expressions. HNEpCs pre-exposed to tunicamycin $(0.5 \mu \mathrm{g} / \mathrm{mL})$ were treated with different doses of 4-PBA (0, 0.25, 0.5, $1 \mathrm{mM})$; an ER stress inhibitor. RT-PCR and real-time PCR showed that 4-PBA significantly attenuated the tunicamycin-induced expressions of MUC5AC and MUC5B mRNA (Fig. 1E-G), ELISA also showed that 4-PBA significantly attenuated the tunicamycin-induced productions of MUC5AC and MUC5B glycoproteins (Fig. 1H).
The three major signaling mechanisms were involved in the ER stress-induced expressions of MUC5AC and MUC5B in HNEpCs

To investigate the roles of three major signaling pathways, that is, the IRE1/XBP-1, PERK/ATF4/CHOP, and the ATF6 pathways, on the expressions of MUC5AC and MUC5B in HNEpCs, cells were stimulated with different doses of tunicamycin with/without 4-PMA for 1 hour, and the mRNA expressions of spliced XBP-1, CHOP, and ATF6 were then checked. RT-PCR and realtime PCR showed that their mRNA expressions were significantly increased at all tunicamycin doses $(0.25,0.5,1,2.5 \mu \mathrm{g} / \mathrm{mL})$ (Fig. 2A, B) and attenuated by 4-PBA. (Fig. 2C, D). Western blot was also performed to check the levels of XBP-1, CHOP, and ATF6. The results showed that tunicamycin induced XBP-1,

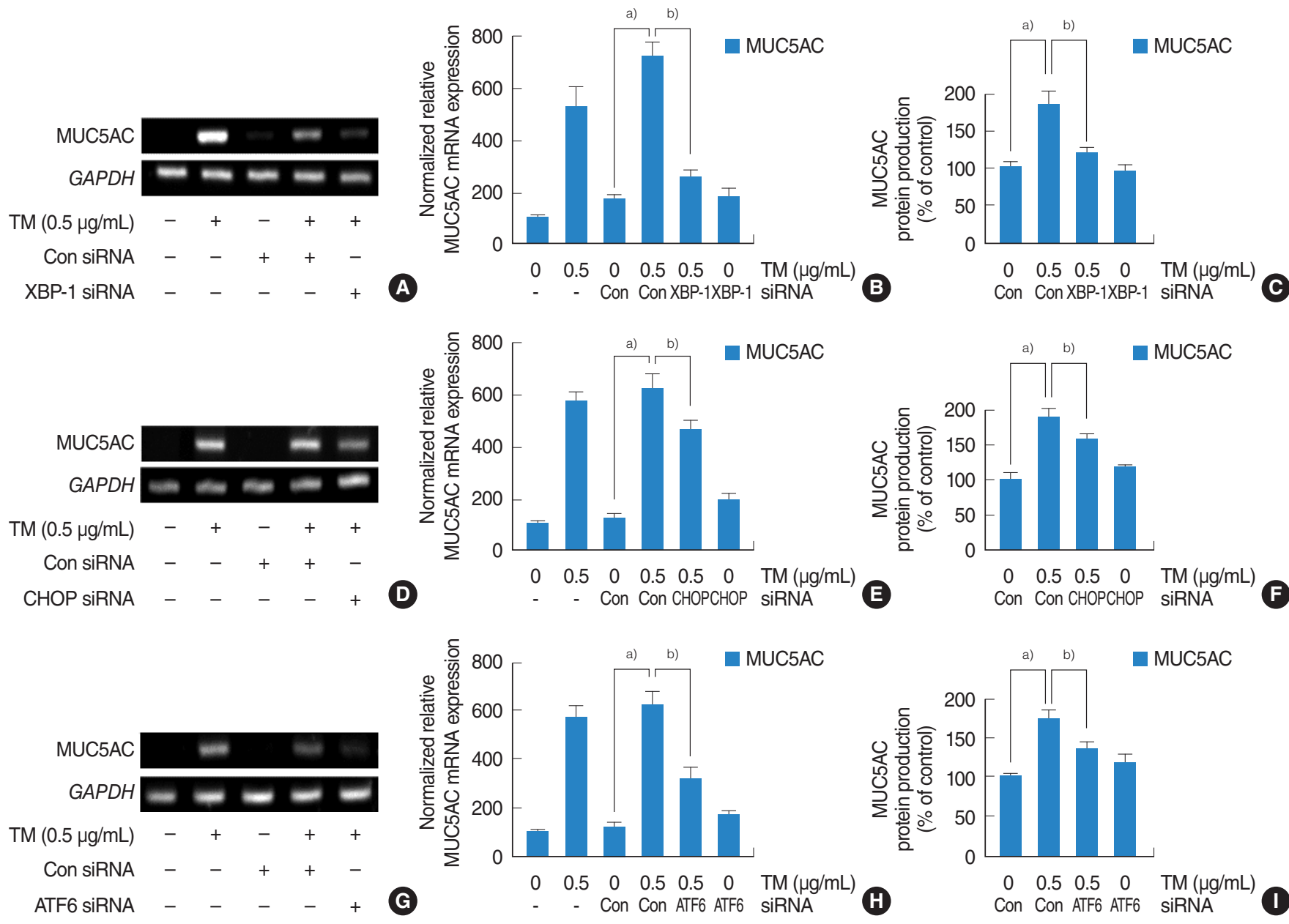

Fig. 3. Contributions of the three major pathways to the upregulation of MUC5AC expression by endoplasmic reticulum stress in human nasal epithelial cells. (A-I) Reverse transcriptase-polymerase chain reaction (RT-PCR), real-time PCR and enzyme-linked immunosorbent assay showed that small interfering RNA (siRNA) knockdowns of XBP-1, CHOP, or ATF6 significantly blocked the endoplasmic reticulum stress-induced MUC5AC mRNA expression and glycoprotein production. (A-C) Inhibition by XBP-1 siRNA was greatest. (D-I) Inhibitions by CHOP and ATF6 siRNA were limited, and inhibition by CHOP siRNA was weakest. The images shown are representative of three separate experiments performed in triplicate. Bars represent the averages \pm standard deviation of three independent experiments performed in triplicate. GAPDH, glyceraldehyde-3-phosphate dehydrogenase; TM, tunicamycin; XBP-1, X-box binding protein 1; Con, control; CHOP, CCAAT-enhancer-binding protein homologous protein; ATF6, activating transcription factor 6 . $\left.{ }^{a}\right) P<0.05$ vs. control siRNA. b) $P<0.05$ vs. TM $(0.5 \mu \mathrm{g} / \mathrm{mL})$ with control SiRNA. 
CHOP and ATF6 and attenuated by 4-PBA (Fig. 2E-G). In addition, transfection with siRNAs for XBP-1, CHOP, and ATF6 were performed to evaluate individual pathway involvements. RT-PCR, real-time PCR and ELISA were used to assess the contributions of the three major mechanisms to the ER stress-mediated expressions of MUC5AC and MUC5B. RT-PCR, real-time PCR and ELISA showed that siRNA knockdowns of XBP-1, CHOP, or ATF6 significantly blocked ER stress-induced MUC5AC mRNA expression and glycoprotein production (Fig. 3) although inhibition by XBP-1 siRNA was greatest (Fig. 3A-C), and inhibition by CHOP siRNA was weakest (Fig. 3D-F). In the study on MUC5B expression, all three siRNAs significantly blocked ER stress-induced MUC5B mRNA expression and glycoprotein production (Fig. 4). However, the inhibitory pattern differed from that of MUC5AC. In this case, inhibition by CHOP siRNA was the greatest (Fig. 4D-F), and inhibitions by XBP-1 was weakest (Fig. 4A-C).

\section{DISCUSSION}

ER is an organelle found in eukaryotic cells, which serves multiple functions, being important particularly in the biosynthesis, processing, and transport of proteins and lipids. ER stress is a response to mismatched protein folding, which lead by an accumulation of misfolded or unfolded proteins in ER lumen [1,2]. ER stress activates three ER transmembrane receptors, that is, IRE1, PERK, and ATF6, to maintain ER homeostasis [15]. ER
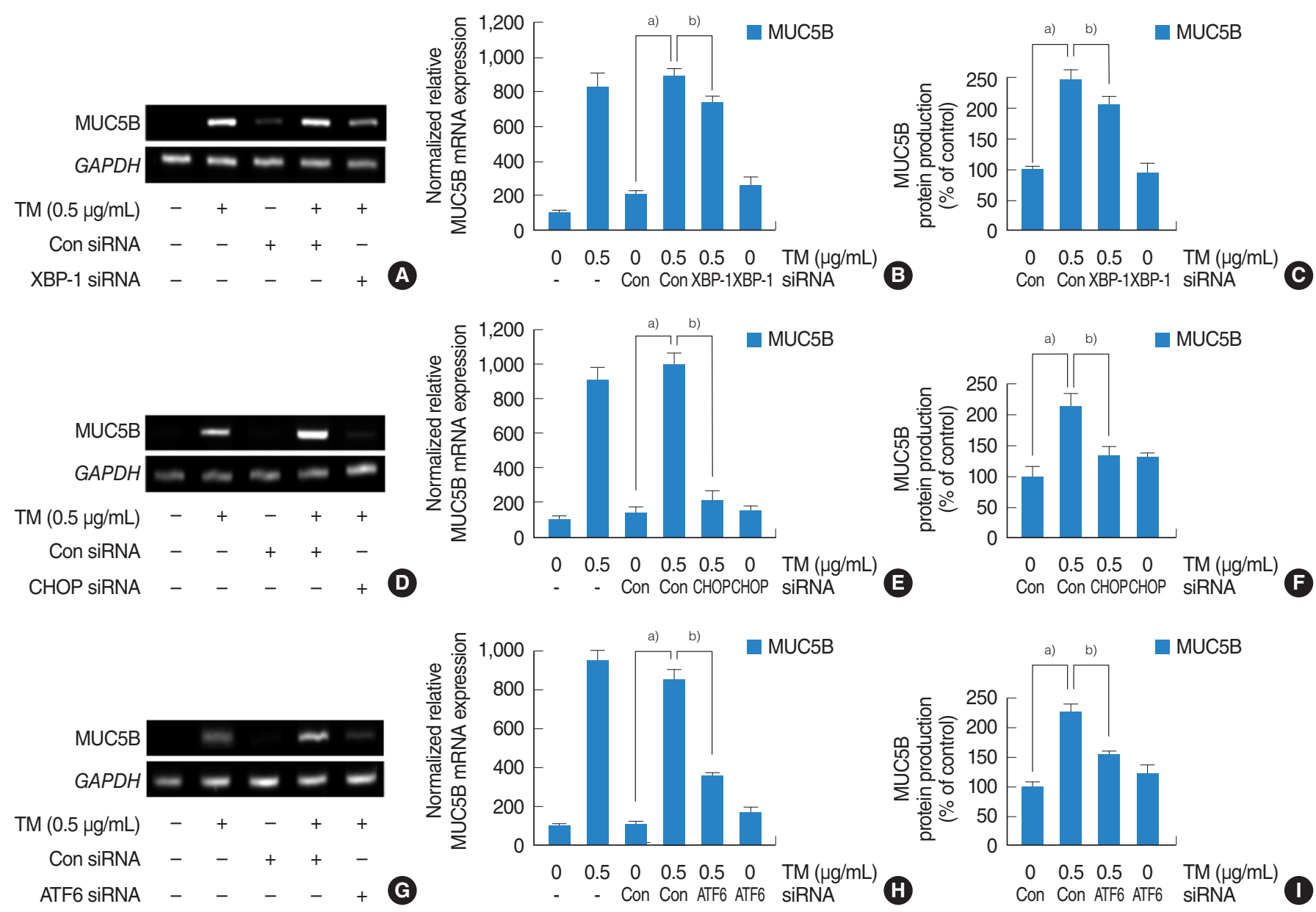

Fig. 4. Contributions of the three major pathways to the upregulation of MUC5B expression by endoplasmic reticulum stress in human nasal epithelial cells. (A-I) Reverse transcriptase-polymerase chain reaction (RT-PCR), real-time PCR and enzyme-linked immunosorbent assay showed that small interfering RNA (siRNA) knockdowns of XBP-1, CHOP, or ATF6 significantly blocked the endoplasmic reticulum stress-induced MUC5B mRNA expression and glycoprotein production. (D-F) Inhibition by CHOP siRNA was greatest. (A-C, G-I) Inhibitions by XBP-1 and ATF6 siRNA were limited, and inhibition by XBP-1 siRNA was weakest. The images shown are representative of three separate experiments performed in triplicate. Bars represent the averages \pm standard deviation of three independent experiments performed in triplicate. GAPDH, glyceraldehyde-3-phosphate dehydrogenase; TM, tunicamycin; Con, control; XBP-1, X-box binding protein 1; CHOP, CCAAT-enhancer-binding protein homologous protein; ATF6, activating transcription factor 6 . ${ }^{\text {a) }} P<0.05 \mathrm{vs}$. control siRNA. ${ }^{\text {b) }} P<0.05 \mathrm{vs}$. TM $(0.5 \mu \mathrm{g} / \mathrm{mL}$ ) with control siRNA. 
stress reduces protein synthesis via the activation of PERK-eukaryotic initiation factor 2 alpha (eIF2 $\alpha$ ), but increases XBP-1and ATF6-mediated chaperone transcription to attenuate ER stress. However, when ER stress persists, ER stress induces apoptosis by activating the PERK-ATF4-CHOP signal pathway $[15,16]$.

ER stress is closely associated with the pathogeneses of various diseases including diabetes, obesity, cancer, autoimmune diseases, and inflammatory airway and bowel diseases [1,2,17]. Moreover, it has been reported in recent studies that ER stressmediated inflammatory reaction in the human airway plays an important role in the initiation, progression, or deterioration of most inflammatory airway diseases, including chronic obstructive airway disease, asthma, cystic fibrosis, and chronic sinusitis with nasal polyposis [3,4,18-21]. Cigarette smoke, as a major cause of chronic airway diseases, induces ER stress by activating the PERK-eIF2 $\alpha$ pathway [18]. In addition, the pathogenesis of bronchial asthma, especially severe steroid-resistant asthma is also associated with the activation of NF- $\mathrm{KB}$ mediated by ER stress $[3,19]$. Spliced XBP-1 mRNA mediated by ER stress is responsible for $\mathrm{ER} / \mathrm{Ca}^{2+}$ store expansion in inflamed human bronchial epithelial cells [20]. In the recent study by Kim et al. [21], they reported that Staphylococcus aureus enterotoxin B (SEB) induced ER stress via reactive oxygen species production in airway epithelial cells. And the induction of unfolded protein response by SEB was increased significantly in eosinophilic polyp compared with noneosinophilic polyp or control mucosa. Through these results, they suggested that SEB-induced ER stress may play important roles in the pathogenesis of nasal polyposis. Furthermore, recent studies indicate that ER is involved in the production of airway mucins: The ER-resident protein anterior gradient homolog 2 (AGR2) increases with mucin overproduction in individuals with asthma and in asthma mouse models. AGR2 interact with immature mucin in the ER and loss of AGR2 impairs allergen-induced MUC5AC and MUC5B overproduction. This study suggested that AGR2 is required for production of the gel-forming airway mucin MUC5AC and MUC5B [22]. And, in the study by Martino et al. [5], it has identified that the ER stress transducer IRE1 $\beta$ is expressed in airway mucous cells and functionally required for the production of airway mucins. Thus, it has been shown that ER is an important organelle in the regulation of the production of airway mucins and ER stress-mediated signaling pathway is also deeply involved in the production of airway mucins. However, the precise involved mechanisms are not fully understood yet. The results of the present study demonstrate that the overproduction of the major secretary airway mucins including MUC5AC and MUC5B, which observed in CRS, are mediated by tunicamycin (an ER stress inducer), and that the tunicamycin-mediated overproductions of MUC5AC and MUC5B can be attenuated by 4-PBA (an ER stress inhibitor).

Increased secretions of MUC5AC and MUC5B glycoproteins are observed from most epithelia affected by inflammatory airway disease $[8-10,23,24]$. But, the specific mechanisms responsible for the up- or down-regulations of the productions of MUC5AC or MUC5B are unclear. According to recent animal studies conducted by Roy et al. [10] on MUC5AC- or MUC5B-deficient or overexpressing mice, the functional roles of these two mucin glycoproteins differ in the mouse respiratory system. It was suggested mouse MUC5B is required for controlling infections in airways and the middle ear, and for maintaining immune homeostasis in mouse lungs, whereas MUC5AC is ineffective. Thus, based on the findings of this previous study, it was expected the productions of MUC5AC and MUC5B would be regulated by different mechanisms. For this reason we focused individually of the involvements of XBP-1, CHOP, and ATF6 in the ER stress-mediated overproductions of MUC5AC and MUC5B in human airway epithelial cells. It was found that all three major ER stress-mediated signaling pathways were involved in the ER stress-mediated overproductions of MUC5AC and MUC5B and that the three pathways were involved individually in the up-regulations of MUC5AC and MUC5B in human airway epithelial cells. In addition, we confirmed that the contributions of the three major pathways to the ER stress-mediated expressions of MUC5AC and MUC5B differed, that is, XBP-1 and ATF6 were mainly involved in ER stress-mediated MUC5AC expression and CHOP and ATF6 were mainly involved in ER stress-mediated MUC5B expression.

The present study has some limitations that should be mentioned. First, assessments of the activation statuses of signaling molecules generated during ER stress were insufficient. Second, due to the lack of further experimental confirmations by using with animal model or human tissue samples, we could not identify the usefulness of clinical application of ER stress-mediated substances as a therapeutic target or biomarker in human sinonasal diseases. Therefore, we suggest additional experiments be conducted to clarify the results of the present study.

In conclusion, the present study demonstrates that ER stress induces the expressions of MUC5AC and MUC5B via the activations of XBP-1, CHOP, and ATF6. And these three major ER stress-mediated signaling networks were found to be differently involved in ER stress-mediated overproductions of MUC5AC and MUC5B in human nasal epithelial cell. These novel findings suggest that precise and specific inhibitions of the three major ER stress signaling pathways offer an effective strategy for developing therapeutics to treat chronic inflammatory sinonasal diseases.

\section{CONFLICT OF INTEREST}

No potential conflict of interest relevant to this article was reported. 


\section{ACKNOWLEDGMENTS}

This work was supported by the 2015 Yeungnam University research grant, Daegu, Republic of Korea.

\section{ORCID}

Min Han Kim ～https://orcid.org/0000-0002-4741-5604

Yong-Dae Kim https://orcid.org/0000-0003-0501-966X

\section{REFERENCES}

1. Cao SS, Luo KL, Shi L. Endoplasmic reticulum stress interacts with inflammation in human diseases. J Cell Physiol. 2016 Feb;231(2): 288-94.

2. Hasnain SZ, Lourie R, Das I, Chen AC, McGuckin MA.The interplay between endoplasmic reticulum stress and inflammation. Immunol Cell Biol. 2012 Mar;90(3):260-70.

3. Kim SR, Lee YC. Endoplasmic reticulum stress and the related signaling networks in severe asthma. Allergy Asthma Immunol Res. 2015 Mar;7(2):106-17.

4.Wei J, Rahman S, Ayaub EA, Dickhout JG, Ask K. Protein misfolding and endoplasmic reticulum stress in chronic lung disease. Chest. 2013 Apr;143(4):1098-105.

5. Martino MB, Jones L, Brighton B, Ehre C, Abdulah L, Davis CW, et al. The ER stress transducer IRE1 $\beta$ is required for airway epithelial mucin production. Mucosal Immunol. 2013 May;6(3):639-54.

6. Mijosek V, Lasitschka F, Warth A, Zabeck H, Dalpke AH, Weitnauer M. Endoplasmic reticulum stress is a danger signal promoting innate inflammatory responses in bronchial epithelial cells. J Innate Immun. 2016 Aug;8(5):464-78.

7. Delmotte P, Sieck GC. Interaction between endoplasmic/sarcoplasmic reticulum stress (ER/SR stress), mitochondrial signaling and $\mathrm{Ca}(2+)$ regulation in airway smooth muscle (ASM). Can J Physiol Pharmacol. 2015 Feb;93(2):97-110.

8. Ali MS, Pearson JP. Upper airway mucin gene expression: a review. Laryngoscope. 2007 May;117(5):932-8.

9. Rose MC, Voynow JA. Respiratory tract mucin genes and mucin glycoproteins in health and disease. Physiol Rev. 2006 Jan;86(1):24578.

10. Roy MG, Livraghi-Butrico A, Fletcher AA, McElwee MM, Evans SE, Boerner RM, et al. Muc5b is required for airway defence. Nature.
2014 Jan;505(7483):412-6.

11. Ha EV, Rogers DF. Novel therapies to inhibit mucus synthesis and secretion in airway hypersecretory diseases. Pharmacology. 2016 Jan;97(1-2):84-100.

12. Voynow JA, Rubin BK. Mucins, mucus, and sputum. Chest. 2009 Feb; 135(2):505-12.

13. Song SY, Woo HJ, Bae CH, Kim YW, Kim YD. Expression of leptin receptor in nasal polyps: leptin as a mucosecretagogue. Laryngoscope. 2010 May;120(5):1046-50.

14. Kim YD, Bae CH, Song SY, Choi YS. Effect of beta-glucan on MUC4 and MUC5B expression in human airway epithelial cells. Int Forum Allergy Rhinol. 2015 Aug;5(8):708-15.

15. Ron D, Walter P. Signal integration in the endoplasmic reticulum unfolded protein response. Nat Rev Mol Cell Biol. 2007 Jul;8(7):51929.

16. Oyadomari S, Mori M. Roles of CHOP/GADD153 in endoplasmic reticulum stress. Cell Death Differ. 2004 Apr;11(4):381-9.

17. Garg AD, Kaczmarek A, Krysko O, Vandenabeele P, Krysko DV, Agostinis P. ER stress-induced inflammation: does it aid or impede disease progression?Trends Mol Med. 2012 Oct;18(10):589-98.

18. Jorgensen E, Stinson A, Shan L, Yang J, Gietl D, Albino AP. Cigarette smoke induces ndoplasmic reticulum stress and the unfolded protein response in normal and malignant human lung cells. BMC Cancer. 2008 Aug;8:229.

19. Kim SR, Kim DI, Kang MR, Lee KS, Park SY, Jeong JS, et al. Endoplasmic reticulum stress influences bronchial asthma pathogenesis by modulating nuclear factor kappaB activation. J Allergy Clin Immunol. 2013 Dec;132(6):1397-408.

20. Martino ME, Olsen JC, Fulcher NB, Wolfgang MC, O'Neal WK, Ribeiro $\mathrm{CM}$. Airway epithelial inflammation-induced endoplasmic reticulum $\mathrm{Ca} 2+$ store expansion is mediated by $\mathrm{X}$-box binding protein-1. J Biol Chem. 2009 May;284(22):14904-13.

21. Kim YM, Jin J, Choi JA, Cho SN, Lim YJ, Lee JH, et al. Staphylococcus aureus enterotoxin B-induced endoplasmic reticulum stress response is associated with chronic rhinosinusitis with nasal polyposis. Clin Biochem. 2014 Jan;47(1-2):96-103.

22. Schroeder BW,Verhaeghe C, Park SW, Nguyenvu LT, Huang X, Zhen $\mathrm{G}$, et al. AGR2 is induced in asthma and promotes allergen-induced mucin overproduction. Am J Respir Cell Mol Biol. 2012 Aug;47(2): 178-85.

23. Kirkham S, Sheehan JK, Knight D, Richardson PS, Thornton DJ. Heterogeneity of airways mucus: variations in the amounts and glycoforms of the major oligomeric mucins MUC5AC and MUC5B. Biochem J. 2002 Feb;361(Pt 3):537-46.

24. Morcillo EJ, Cortijo J. Mucus and MUC in asthma. Curr Opin Pulm Med. 2006 Jan;12(1):1-6. 